\title{
Blood Chromogranin A Is Not Effective as a Biomarker for Diagnosis or Management of Bronchopulmonary Neuroendocrine Tumors/Neoplasms
}

\author{
Somer Matar ${ }^{a}$ Anna Malczewskab, c Kjell Oberg ${ }^{d}$ Lisa Bodei $^{\mathrm{e}}$ \\ Harry Aslanian $^{b}$ Anna Lewczuk-Myślicka ${ }^{f}$ Pier Luigi Filosso $^{9}$ \\ Alejandro L. Suarez ${ }^{b}$ Agnieszka Kolasińska-Ćwikła ${ }^{\text {h }}$ Matteo Roffinellag \\ Beata Kos-Kudłac Jarosław B. Ćwikłai Ignat A. Drozdova Mark Kidd ${ }^{\mathrm{a}}$ \\ Irvin M. Modlin ${ }^{\mathrm{j}}$
}

\begin{abstract}
${ }^{a}$ Wren Laboratories, Branford, CT, USA; bSection of Digestive Diseases, Yale University School of Medicine, New Haven, CT, USA; ' Department of Endocrinology and Neuroendocrine Tumors, Medical University of Silesia, Katowice, Poland; ${ }^{d}$ Department of Endocrine Oncology, University Hospital, Uppsala, Sweden; ${ }^{\text {eDepartment }}$ of Radiology, Memorial Sloan Kettering Cancer Centre, New York, NY, USA; ${ }^{\circledR}$ Department of Endocrinology and Internal Medicine, Medical University of Gdańsk, Gdańsk, Poland; ${ }^{9}$ Department of Thoracic Surgery, University of

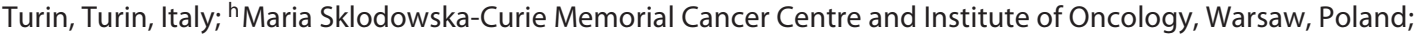
'Department of Radiology, University of Warmia and Mazury, Olsztyn, Poland; ${ }^{j}$ Gastroenterological and Endoscopic Surgery, Yale University School of Medicine, New Haven, CT, USA
\end{abstract}

\section{Keywords}

Bronchopulmonary · Lung · Chromogranin A · Biomarker · Carcinoid · Diagnosis · Prognosis

\begin{abstract}
Background: Identification of circulating tumor markers for clinical management in bronchopulmonary (BP) neuroendocrine tumors/neoplasms (NET/NEN) is of considerable clinical interest. Chromogranin A (CgA), a "universal" NET biomarker, is considered controversial as a circulating biomarker of BPNEN. Aim: Assess utility of $\mathrm{CgA}$ in the diagnosis and management of BPNEN in a multicentric study. Material and Methods: CgA diagnostic metrics were assessed in lung NET/ NENs ( $n=200)$ and controls $(n=140)$, randomly assigned to a Training and Test set (100 BPC and 70 controls in each). As-
\end{abstract}

\section{KARGER}

(c) 2019 S. Karger AG, Basel

E-Mail karger@karger.com

www.karger.com/nen say specificity was evaluated in neoplastic lung disease ( $n=$ $137)$ and nonneoplastic lung disease $(n=77)$. CgA efficacy in predicting clinical status was evaluated in the combined set of 200 NET/NENs. CgA levels in bronchopulmonary neuroendocrine tumor (BPNET) subtypes (atypical [AC] vs. typical [TC]) and grade was examined. The clinical utility of an alteration of $\mathrm{CgA}$ levels ( $\pm 25 \%$ ) was evaluated in a subset of 49 BPNET over 12 months. CgA measurement was by NEOLISA ${ }^{\text {TM }}$ kit (EuroDiagnostica). Results: Sensitivity and specificity in the training set were $41 / 98 \%$, respectively. Test set data were $42 / 87 \%$. Training set area under receiver operator characteristic analysis differentiated BPC from control area under the curve (AUC) $0.61 \pm 0.05 p=0.015$. Test set the data were AUC $0.58 \pm 0.05, p=0.076$. In the combined set $(n=200), 67 \%$ BPNET/NEN ( $n=134$ ) had normal CgA levels. CgA levels did not distinguish histological subtypes (TC vs. AC, AUC $0.56 \pm$ 
$0.04, p=0.21)$, grade $(p=0.45-0.72)$, or progressive from stable disease (AUC $0.53 \pm 0.05 p=0.47$ ). There was no correlation of CgA with Ki-67 index (Pearson $r=0.143, p=0.14$ ). For nonneoplastic diseases (chronic obstructive pulmonary disorder and idiopathic pulmonary fibrosis), CgA was elevated in 26-37\%. For neoplastic disease (NSCLC, squamous cell carcinoma), $\mathrm{CgA}$ was elevated in $11-16 \%$. The neuroendocrine SCLC also exhibited elevated CgA (50\%). Elevated CgA was not useful for differentiating BPNET/NEN from these other pathologies. Monitoring BPNET/NEN over a 12-month period identified neither $\mathrm{CgA}$ levels per se nor changes in $\mathrm{CgA}$ were reflective of somatostatin analog treatment outcome/efficacy or the natural history of the disease (progression). Conclusions: Blood CgA levels are not clinically useful as a biomarker for lung BPNET/NEN. The low specificity and elevations in both nonneoplastic as well as other common neoplastic lung diseases identified limited clinical utility for this biomarker.

(c) 2019 S. Karger AG, Basel

\section{Introduction}

Cancer biomarkers are essential for the identification of disease and as tools to quantify disease progression (PD) and outcome. Numerous types of biomarkers have been proposed and studied [1]. Among these, dysregulated physiological processes such as increased secretory products have been investigated since they represent a process specific to a cell type and are quantifiable, thereby providing a reflection of disease status or a surrogate for treatment efficacy [2]. The acquisition of biological fluids (e.g., urine, saliva, tears, and plasma) is less invasive than tissue biopsy and all possess assessable analytes ranging from nucleic acids to tumor cells [3]. Irrespective of the diverse biomarkers available, a critical issue in assessing clinical utility is the need for rigorous establishment of diagnostic sensitivity and specificity [4]. A further metric of particular clinical relevance is the demonstration that alterations in biomarker levels can be correlated with clinical management of disease. Overall, imaging and blood biomarker measurements remain the 2 main strategies for monitoring disease status. The former, however, requires sophisticated equipment, often includes exposure to radiation and in some relatively indolent diseases, such as neuroendocrine tumors (NET), has limitations in sensitivity [5].

Bronchopulmonary neuroendocrine tumors (BPNET: also known as carcinoids) are neuroendocrine neoplasms (NEN) that range from low-grade typical (TC) to intermediate-grade atypical (AC) tumors [6]. The primary diag- nosis for BPNET/NEN is by histological analysis of tissue obtained by surgery or biopsy [7]. Identification of a neuroendocrine phenotype relies on the elucidation of tumor architectural features and immunohistochemical markers such as chromogranin A (CgA), synaptophysin, and CD56 [6]. Assessment of the rate of tumor cell proliferation is, to some extent, defined by Ki-67 immunostaining [8]. Differentiation of histological variants, TC from AC, is undertaken by mitotic count: $\mathrm{TC}<2$ mitoses/ 10 high power field, AC 2-10 mitoses/10 high power field $[6,8]$. In addition, the presence of necrotic features in AC tumors compared to TC is considered to be of value in distinguishing the groups. Imaging is of some benefit in defining the disease. Thus, CT, MRI ${ }^{68}$ Gallium-DOTATE/TOC/NOC $\mathrm{PET} / \mathrm{CT}$, and FDG PET/CT have been used with varying degrees of success $[9,10]$. Irrespective of grade, surgical resection is the treatment of choice and considered as the definitive curative approach [11]. Postresection disease monitoring is variously undertaken using both blood $\mathrm{CgA}$ measurement and imaging [12].

$\mathrm{CgA}$, a member of the granin family, is a protein in the secretory granules of neuroendocrine cells, which is implicated in the synthesis of signaling molecules [13]. There are a number of CgA-derived peptides, such as pancreastatin and catestatin, involved in the secretory regulation of various hormones as well as metabolic regulation and vasoconstriction [14]. The use of CgA as an immunohistochemical marker is widespread and accepted and is utilized in diverse endocrine diseases [15]. As a circulating biomarker, $\mathrm{CgA}$ can be measured in serum or plasma and is commonly used in assisting the diagnosis and management of gastroenteropancreatic neuroendocrine tumors (GEP-NETs) [16]. Despite its common usage, the specificity of $\mathrm{CgA}$ is $<50 \%$, and the sensitivity in GEP-NETs has been variously reported to range from 60 to $90 \%$ [5]. Further concerns include the widely differing individual assay metrics and spurious results associated with the use of pharmacological agents (proton pump inhibitors) and diverse pathological conditions, especially renal disease $[17,18]$.

Although BPNET/NEN share many homologies with GEP-NET, including symptomology and the secretion of bioactive peptides, a recent NET Biomarker Consensus concluded that GEP-NET circulating biomarkers (i.e., $\mathrm{CgA}$ ) were neither sensitive nor specific for bronchopulmonary neuroendocrine neoplasia [5]. A number of investigations have evaluated circulating CgA in BPNET/ NEN with varying stages of disease and found no significant differences corresponding with nodal metastasis and tumor size following TNM classification [19]. No finite 
assessment of the clinical utility of CgA in BPNET/NEN has yet been undertaken.

It is generally accepted that circulating $\mathrm{CgA}$ exhibits poor diagnostic metrics in NETs [20]. The Consensus on Biomarkers for NETs [5] considers it as a monoanalyte and a class/type "0" according to the NIH biomarker classification. Based upon the concerns relating to the low sensitivity and specificity of CgA as a biomarker in GEPNETs and the minimal studies conducted with large cohorts of BPNET/NEN, we undertook a more robust evaluation of circulating $\mathrm{CgA}$. The aim of this study was to assess the clinical value of circulating $\mathrm{CgA}$ measurement in a comprehensive cohort of healthy controls, BPNET/ NEN with varying histology, grade, status and disease extent, nonneuroendocrine lung diseases, and in BPNET/ NEN patients monitored over time.

\section{Subjects and Methods}

\section{Patients}

All patients provided informed consent for plasma biomarker analysis authorized by local Ethics Committees. This multicenter retrospective cohort was collected between June 2013 and February 2018. It included patients and nonaffected family members attending oncology, endocrinology, and pulmonology out-patient clinics. Inclusion criteria included histological confirmation of disease. No exclusion criteria were used. Cohorts included healthy controls, BPNET/NEN of various grade, histology, status, and therapies, as well as nonneuroendocrine lung diseases (Table 1). Whole-blood plasma for CgA measurement was collected in EDTA-plasma preparation tubes.

The clinical utility of CgA was evaluated in 4 areas: histology, $\mathrm{PD}$, efficacy of somatostatin analogs (SSA), and as a prognostic biomarker. First, we examined the relationship between histological subtype, Ki-67, and CgA levels. For this, we compared CgA plasma levels in TC and AC and evaluated whether these correlated with $\mathrm{Ki}-67$ expression in tumor tissue (data from clinical records). Because this was a multicentric study, no central review was undertaken. Histological assessment at each institution was per standard European Neuroendocrine Tumour Society criteria [12]. We then evaluated its use as a clinical monitoring tool in longitudinally followed patients $(n=49)$. Subjects underwent testing at baseline and thereafter every 3 months for a total of 12 months. Second, the relationship between $\mathrm{CgA}$ and clinical status was examined. This was assessed by imaging every 3 months and disease stabilization (SD) or PD determined by RECIST 1.0 criteria in the 49 -patient cohort. This allowed us to determine whether CgA provided a real-time measurement that accurately correlated with disease status (disease stabilization [SD] or PD). Third, we evaluated the effect of SSAs on CgA levels. A subset of twenty patients ( $41 \%$ of the 49) was treated with Sandostatin LAR (20 or $30 \mathrm{mg}$ ), and $\mathrm{CgA}$ levels were evaluated in terms of response to therapy. Fourth, we examined whether CgA had use as a type 0 NIH biomarker (a marker of the natural history of disease or prognostic marker, and whether it correlated with clinical outcomes - SD or PD).

\section{Disease Cohorts}

We examined circulating CgA levels from BPNET/NEN $(n=$ $200)$ compared to healthy controls $(n=140)$, benign pulmonary disease: idiopathic pulmonary fibrosis (IPF: $n=50$ ), chronic obstructive pulmonary disorder (COPD: $n=27)$; neoplastic lung disease: small cell lung cancer (SCLC: $n=32$ ), squamous cell carcinoma (SCC: $n=43$ ), and adenocarcinomas cell carcinoma (ACC: $n=62)$.

\section{Analytic Strategy}

All plasma samples were randomly assigned to a training and test group. Each of these 2 sets comprised 100 carcinoids and 70 controls. Once all samples were measured, an independent correlation of each value with the clinical diagnosis was undertaken. Diagnostic accuracy and metrics (sensitivity, specificity) were calculated for each set. The area under the curve (AUC) and $p$ value were then calculated between various cohorts (non-NE lung diseases, histological variants and grade, and in clinical status). For clinical utility, we correlated changes in CgA levels with disease outcome.

\section{CgA Enzyme-Linked Immunosorbent Assay}

CgA was measured using the NEOLISA ${ }^{\mathrm{TM}} \mathrm{CgA}$ kit (EuroDiagnostica, Malmo, Sweden). The assay was undertaken in a clinical laboratory (Wren Laboratories CL-0704, CLIA 07D2081388). The upper limit of normal for this CgA ELISA assay is $\leq 108 \mathrm{ng} / \mathrm{mL}$ [21].

\section{Statistical Analysis}

D'Agostino and Pearson normality tests and the Shapiro-Wilk normality test both identified that CgA was not normally distributed (K2: 59.07-145.1; W-value: 0.257-0.73; both $p<0.0001$ ). We therefore used nonparametric statistics for analyses [22]. Nonparametric (Mann-Whitney U test, 2-tailed) comparisons, Fisher's exact test (2-tailed), and receiver operator characteristic curve analyses were therefore used between cohorts. In one instance, the relationship between 3 categorical variables was tested using a 2-tailed $\chi^{2}$ test. Metrics calculated included sensitivity, specificity, the $z$ score (values $>1.96$ are significant), calculation of the Youden J index (performance of a diagnostic), Kaplan-Meier survival analysis, and spider plots. For spider plot analyses, a 2-way ANOVA (using Sidak's multiple corrections test) was undertaken to evaluate differences between means in each of the 2 categories (treated vs. untreated) at the different time points. Prism 6.0 for Windows (GraphPad Software, La Jolla, CA, USA, www.graphpad.com) and MedCalc Statistical Software version 16.2.1 (MedCalc Software bvba, Ostend, Belgium; http://www.medcalc.org; 2017) were utilized. Values are provided as mean $\pm \mathrm{SD}$ and (median [interquartile range]).

\section{Results}

\section{A. Diagnostic Utility}

A1 Discovery Set: BPNETs/NENs and Controls

The initial study established circulating CgA plasma levels in a Training Set and thereafter in a Test Set. CgA levels in BPNET/NEN were compared with controls and the metrics for each set defined utilizing the NEOLISA ${ }^{\mathrm{TM}}$ $108 \mathrm{ng} / \mathrm{mL}$ cutoff. 


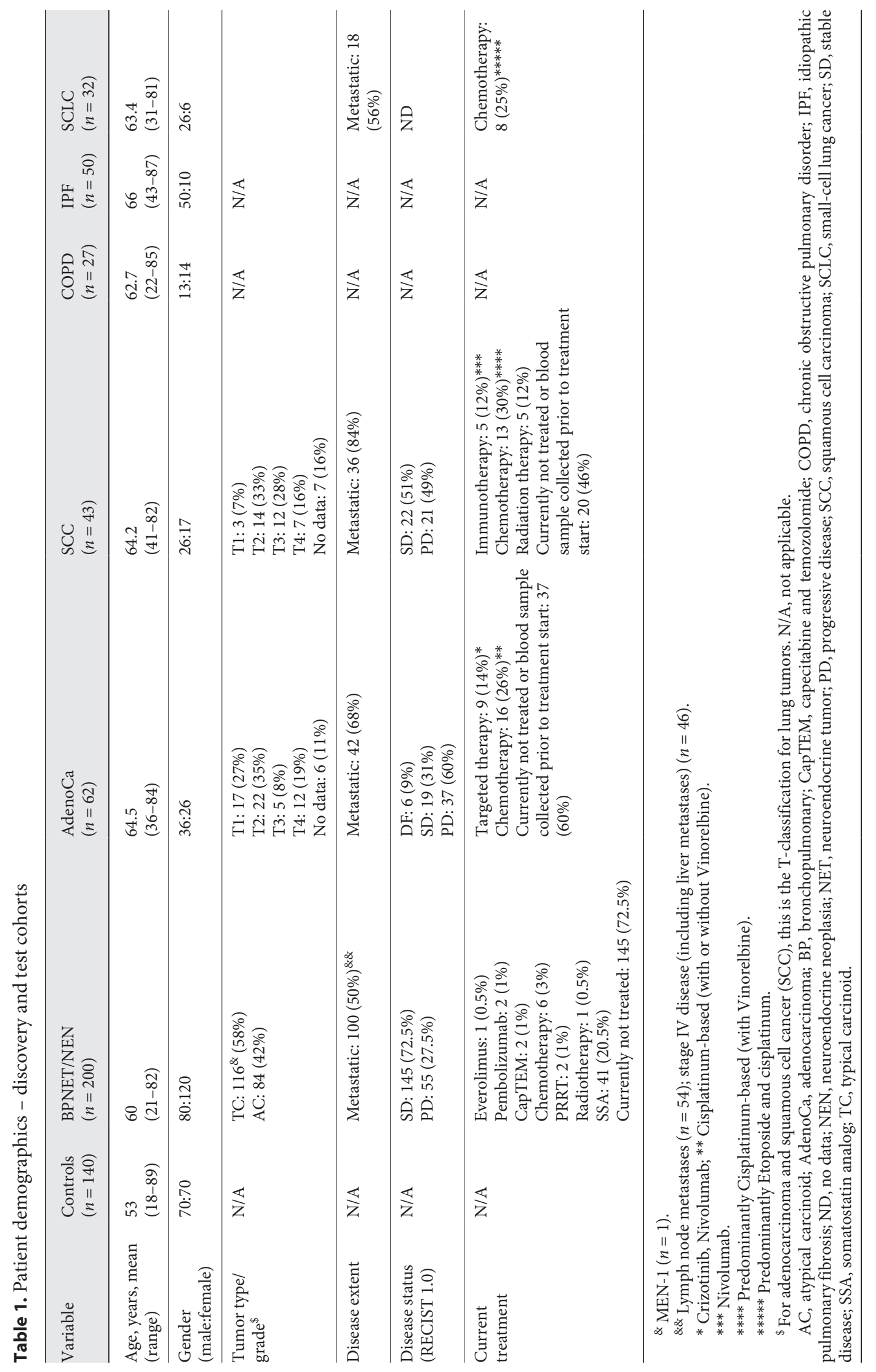




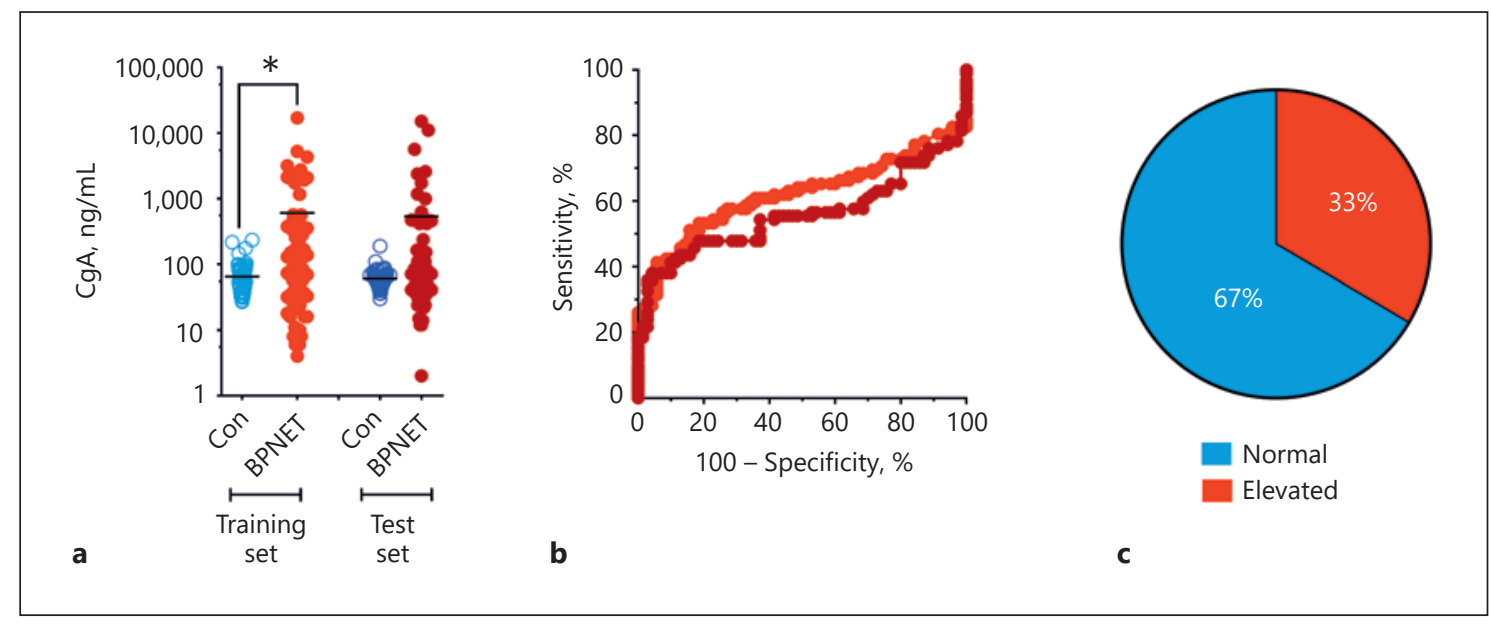

Fig. 1. CgA levels in BPNET/NEN training and test sets. a Training set CgA levels: In BPNET/NEN, mean level was elevated versus controls $(* p<0.01)$. In the test set, mean BPNET/NEN level was not different to controls $(p=0.2)$. b The AUC for differentiating BPNET/NEN from controls for the training set was 0.61 (95\% CI
$0.53-0.69, p=0.015)$ and for the test set was 0.58 (95\% CI 0.50 $0.66, p=0.076)$. c CgA levels were elevated in $33 \%(n=66)$ of the 200 BPNET/NEN. The remaining 134 (67\%) samples fell within normal levels ( $\leq 108 \mathrm{ng} / \mathrm{mL})$. CgA, chromogranin A.
A1.1 Training Set. The mean circulating CgA level in BPNET/NEN from the training set $(n=100,604 \pm 1,961$ $\mathrm{ng} / \mathrm{mL} ;[83(35-273)])$ was significantly higher $(p<0.01)$ compared to controls $(n=70,65 \pm 38 \mathrm{ng} / \mathrm{mL}$; [55 (4175)]; Fig. 1a). AUC analysis could differentiate BPNET/ NEN from controls (AUC $0.61 \pm 0.05, p=0.015, z$ score: 2.43, Youden index J: 40\%) but although significant, it should be noted that an AUC of 0.6 would be considered a poor biomarker (Fig. 1b). The sensitivity and specificity for the training set were calculated as 41 and $98 \%$, respectively.

A1.2 Test Set. The mean CgA level was not elevated $(p=0.2)$ in BPNET/NEN from the test set $(n=100,534$ $\pm 2,030 \mathrm{ng} / \mathrm{mL} ;[64(41-107)])$ compared to the controls $(n=70,60 \pm 21 \mathrm{ng} / \mathrm{mL}$; [57 (48-67)]; Fig. 1a). The AUC analysis was not statistically significant (AUC $0.58 \pm 0.05$, $p=0.076, z$ score: 1.78 , Youden index J: $30 \%$; Fig. 1b). The sensitivity and specificity were 42 and $87 \%$ respectively.

A1.3 Combined Set. Mean CgA levels were $569 \pm 1,990$ $\mathrm{ng} / \mathrm{mL}(71$ [38-161]) in the combined set of $200 \mathrm{BPNET} /$ NEN. CgA levels were normal in 67\% $(n=134)$ and elevated in $33 \%(n=66)$ of the cohort (Fig. 1c).

A2 CgA Levels in Comparator Sets: Benign and Malignant Lung Disease

$\mathrm{CgA}$ was evaluated in a cohort of benign lung disease and in nonneuroendocrine malignant lung neoplasia to assess the specificity for BPNET/NEN.

CgA Has No Efficacy in Lung NEN
In the benign cohort, IPF patients $(n=50,103 \pm 114$ $\mathrm{ng} / \mathrm{mL} ;[70(42-121)])$ had an elevated mean value compared to controls $(n=140,62 \pm 31 \mathrm{ng} / \mathrm{mL}$; [57 (46-69)], $p=0.038)$. Twenty-six percent $(n=13)$ of IPF patients had elevated CgA levels compared to $2.8 \%(n=4)$ of controls. This was significant $(p<0.0001$, Fisher's test). Those with COPD also had an increased mean level $(n=27,144$ $\pm 157 \mathrm{ng} / \mathrm{mL}$; [79 (50-182)], $p=0.0037)$ compared to controls (Fig. 2a). The proportion of COPD with elevated CgA levels was 37\% (10/27); this was significant ( $p<$ 0.0001, Fisher's test).

In lung $\mathrm{ACC}$, the mean $\mathrm{CgA}$ level was not elevated $(n=62,60 \pm 30 \mathrm{ng} / \mathrm{mL} ;[56(15-78)], p=0.55$ versus controls); 7 (11\%) exhibited elevated levels. The mean CgA level was marginally elevated in SCC $(n=43,74 \pm 87 \mathrm{ng} /$ $\mathrm{mL}$; [44 (36-68)], $p=0.045$ versus controls); 7 (16\%) were elevated. Both ACC and SCC mean CgA levels were significantly lower than in COPD $(p<0.005)$ but were not different to IPF mean levels ( $p=0.07-0.08)$.

The mean level of CgA in SCLC was significantly elevated $(n=32,118 \pm 83 \mathrm{ng} / \mathrm{mL} ;[106(54-150)])$ versus controls $(62 \pm 31 \mathrm{ng} / \mathrm{mL}$; $[57(46-69)] ; p<0.0001)$ but was not elevated versus COPD $(144 \pm 157 \mathrm{ng} / \mathrm{mL}$; 79 (50$182)] ; p=0.76)$ or IPF $(p=0.11)$. The proportion of SCLC with elevated CgA levels was 50\% (16/32); this was significant ( $p<0.0001$, Fisher's test) versus controls but was not significant versus BPNET/NEN ( $p=0.07$, Fisher's test). 


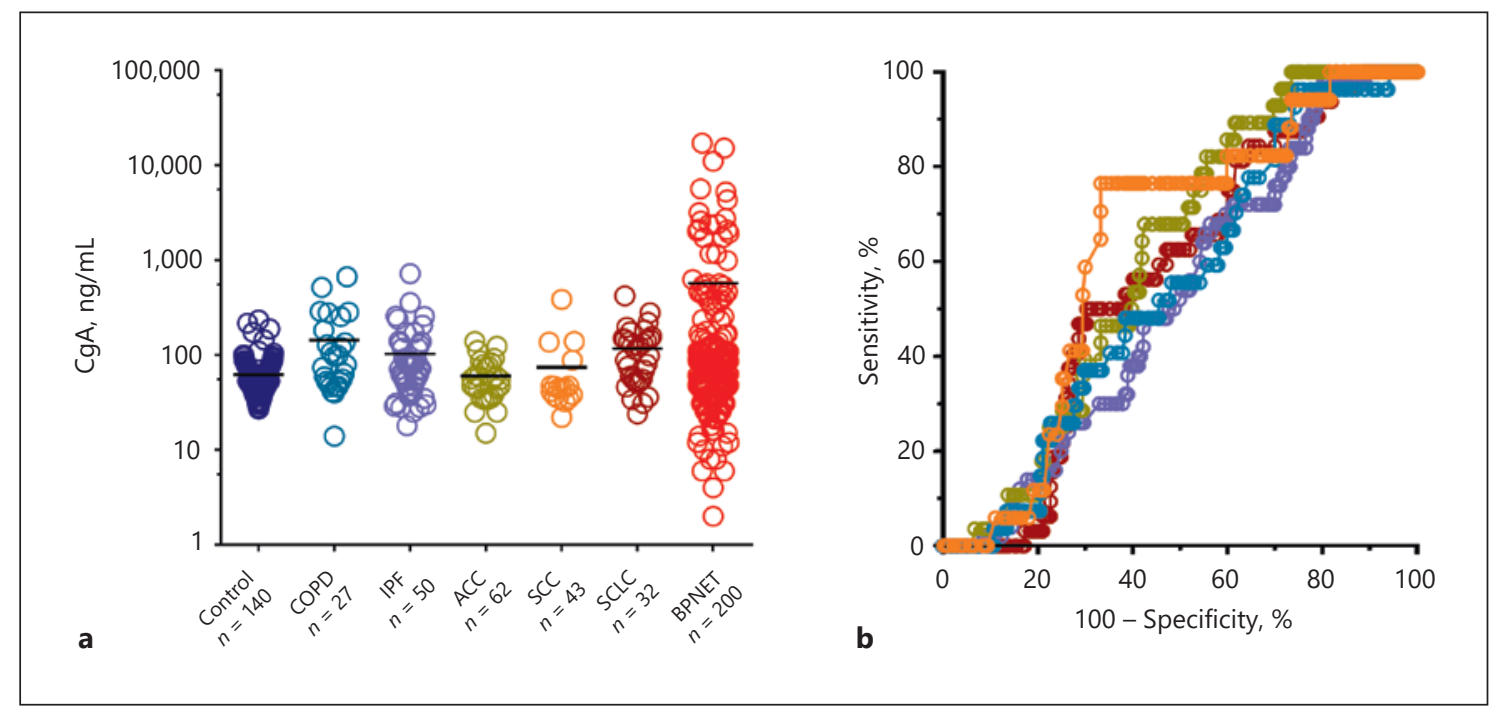

Fig. 2. CgA levels in benign and malignant lung disease. a Mean CgA level in COPD $(n=27)$ was elevated $(p=0.0037)$ compared to controls $(n=140)$. Mean level in ACC $(n=62)$ was not elevated $(p=0.55)$, but the mean level was increased $(p=0.045)$ in SCC $(n=43)$. ACC and SCC CgA levels were significantly less than COPD $(p<0.005)$. IPF $(n=50)$ had elevated mean level compared to controls $(p=0.038)$. SCLC $(n=32)$ also exhibited elevated levels compared to controls $(p<0.0001)$. b AUC analysis could not dif-

AUC analysis could not differentiate between either COPD (AUC $0.54 \pm 0.05, p=0.48$ ) or IPF and BPNET/ NEN (AUC $0.52 \pm 0.04, p=0.64$ ). For ACC (AUC $0.61 \pm$ $0.04, p=0.06)$, SCC (AUC $0.63 \pm 0.06, p=0.06)$, and SCLC (AUC $0.56 \pm 0.05, p=0.23$ ), the metrics were not significant compared to BPNET/NEN (Fig. 2b).

\section{B. Clinical Utility}

B1 CgA Evaluation in BPNET/NEN (TC and AC) and $\mathrm{Ki}-67$

We examined whether CgA levels were associated with histological subtype or correlated with the tissue Ki-67 proliferation index.

B1.1 Histology. CgA levels were evaluated between TC $(n=116)$ and AC $(n=84)$. The mean CgA level was not significantly increased in TC $(415 \pm 1,772 \mathrm{ng} / \mathrm{mL}$; [ 69 $(34-128)]), p=0.11)$ compared to the controls $(n=140$; $62 \pm 31 \mathrm{ng} / \mathrm{mL}$; [57 (46-69)]), but was elevated in AC (672 \pm 2011 ; [72 (47-274)]), $p=0.0041$; Fig. 3a). The proportion of TC with elevated CgA was $35(30 \%)$; this was significant compared to controls $(4 / 140 ; p<0.0001$, Fisher's test). The proportion of $\mathrm{AC}$ with elevated $\mathrm{CgA}$ was 30 (36\%); this was significant compared to controls $(p<$ 0.0001 , Fisher's test). No significant differences in those ferentiate COPD or IPF from BPNET/NEN (AUC 0.52-0.54, $p=$ $0.48-64$ ), and the AUC between ACC and SCC and BPNET/NEN was not statistically significant (AUC $0.61-0.63, p=0.06$ ). SCLC likewise could not be differentiated from BPNET/NEN (AUC 0.56, $p=0.23)$. CgA, chromogranin A; ACC, adenocarcinoma cell carcinoma; COPD, chronic obstructive pulmonary disorder; IPF, idiopathic pulmonary fibrosis; SCC, squamous cell carcinoma; BPNET, bronchopulmonary neuroendocrine tumor.

with an elevated $\mathrm{CgA}$ was noted between $\mathrm{TC}$ and $\mathrm{AC}$ ( $p=0.45$ ). An AUC analysis (TC vs. AC), likewise, was not statistically significant and could not differentiate between the 2 histological variants $(0.56 \pm 0.04, p=0.21$; Fig. 3b).

B1.2 Ki-67. Ki-67 proliferation index data were available in $110(55 \%)$ of the 200 BPNET/NEN. Grade 1 BPNET/NEN ( $n=67, \mathrm{Ki}-67: \leq 4 \%)$ had CgA levels averaging $508 \pm 1,906 \mathrm{ng} / \mathrm{mL}(78[32-178])$ and in Grade 2 BPNET/NEN ( $n=39, \mathrm{Ki}-67: 5-24 \%)$ the levels averaged $943 \pm 2,827 \mathrm{ng} / \mathrm{mL}$ (80 [49-353]). Grade 3 numbers were limited ( $n=4$, Ki-67: $\geq 25 \%)$ and had CgA levels of $188 \pm$ $157 \mathrm{ng} / \mathrm{mL}$ (162 [56-348]). All $p$ values were insignificant ( $p=0.45-0.72$ ) between the 3 grades (Fig. $3 c$ ). No significant relationship could be identified between $\mathrm{CgA}$ levels and Ki-67 values (Pearson $r=0.143, p=0.136$; Fig. $3 \mathrm{~d}$ ). $\mathrm{CgA}$ measurement could not consistently define histological variants or the different grades of BPNET/NEN. CgA did not correlate with the Ki-67 index for BPNET/ NEN.

\section{B2 CgA and Disease Status}

CgA was assessed in the context of stage and clinical status (based on RECIST 1.0 criteria) to determine if lev- 


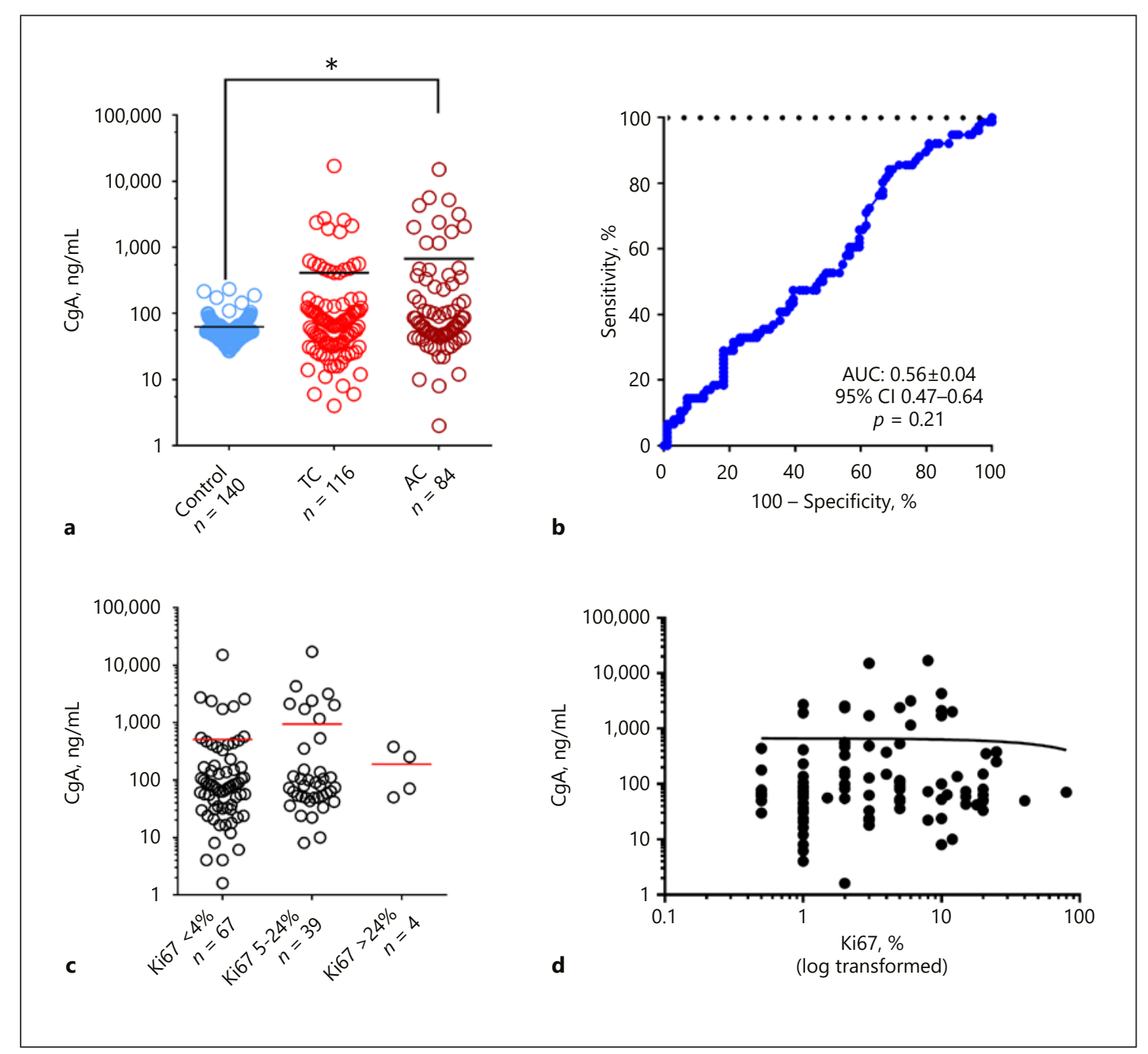

Fig. 3. $\mathrm{CgA}$ measurement in histological variants and grades of BPNET/NEN. a Mean CgA level was significantly elevated $(* p<$ $0.005)$ for AC $(n=84)$ but not TC $(n=116)$, compared with controls $(n=140)$. Both TC and AC had a similar proportion (30 and $36 \%$, respectively) of elevated levels. b AUC analysis could not differentiate CgA levels between TC and AC (AUC 0.56, 95\% CI 0.47$0.64, p=0.21)$. c CgA levels between Grade $1(\mathrm{Ki} 67<4 \% ; n=67)$

els correlated with PD. Patients with liver metastatic disease (Stage IV) comprised 46 (23\%). These patients exhibited an elevated mean CgA level $(2,019 \pm 708 \mathrm{ng} / \mathrm{mL}$; [109 (63-1,723)]) compared to those with no metastasis $(n=100 ; 194 \pm 483 \mathrm{ng} / \mathrm{mL} ;[65(40-106)], p=0.01)$ but not versus those with lymph node disease $(n=54 ; 236 \pm$ $466 \mathrm{ng} / \mathrm{mL}$; [64 (32-209)], $p=0.13)$. The proportion of patients with elevated CgA was 43\% (Stage IV); $33 \%$ (lymph node metastases); and $28 \%$ (no metastases). This was not significantly different $\left(\chi^{2}: p=0.18\right)$.

CgA Has No Efficacy in Lung NEN and Grade 2 (Ki67: 5-24\%; $n=39$ ) BPC were not statistically significant $(p=0.45)$. Grade 3 (Ki67 $>24 \% ; n=4)$ was lower but this was not statistically different $(p=0.56-0.72)$ compared to Grade 1 and 2. d The relationship between Ki-67 grading and CgA levels was not significant (Pearson $r=0.143, p=0.136$ ). CgA, chromogranin A; TC, typical; AC, atypical; AUC, area under the curve.

Patients with PD comprised $28 \%(n=55)$ of the 200 BPNET/NEN cohort, while patients with no image-evidence of disease or stable disease (SD) comprised the remaining cohort $(n=145)$. The PD group did not have a significantly elevated mean CgA level $(401 \pm 769 \mathrm{ng} / \mathrm{mL}$; [70 (46-157)]) compared to SD $(576 \pm 2,184 \mathrm{ng} / \mathrm{mL} ;[70$ (35-152)], $p=0.47)$. However, mean levels in both PD and SD were significantly higher than the mean level in the controls ( $p<0.05$; Fig. 4a). The proportion of SD with elevated CgA was 47 (32\%); this was significant compared 


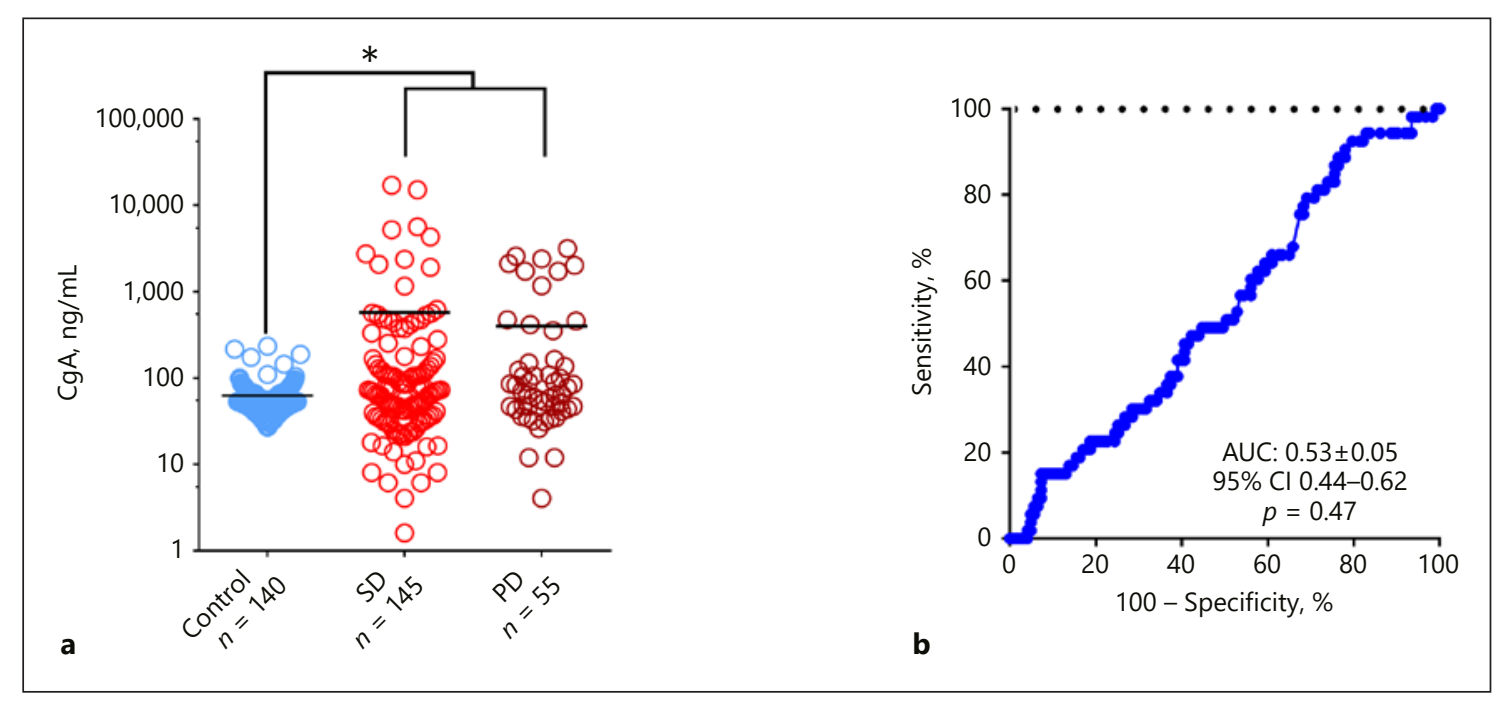

Fig. 4. Assessment of CgA in stable and PD. a Mean CgA level between SD $(n=145)$ and PD $(n=55)$ was not significant $(p=0.47)$. Mean levels in SD and PD were increased $(* p=0.018-0.048)$ compared with controls $(n=140)$. Both SD and PD had a similar pro- portion ( 32 and 31\%, respectively) of elevated levels. b AUC analysis for differentiating SD from PD was not statistically significant (AUC 0.53, 95\% CI 0.44-0.62, $p=0.47$ ). CgA, chromogranin A; $\mathrm{PD}$, progressive disease; AUC, area under the curve. to controls $(4 / 140 ; p<0.0001$, Fisher's test). The proportion of PD with elevated CgA was 17 (31\%); this was significant compared to controls ( $p<0.0001$, Fisher's test). No significant differences in those with an elevated CgA was, however, noted between SD and PD $(p=1.0)$. In addition, AUC analysis could not differentiate progressive disease from stable disease $(0.53 \pm 0.05 p=0.47$; Fig. $4 \mathrm{~b})$.

\section{B3 Clinical Utility of CgA Levels}

Of the 200 BPNET/NEN, only 49 could be studied for clinical utility. Evaluations (clinical, radiological, and CgA occurred every 3-6 months over a 12-month period (Fig. 5). At baseline, 22 (45\%) were clinically characterized as progressive disease, while the remaining 27 (55\%) were identified as clinically stable.

Eight of the 22 patients with progressive disease exhibited elevated CgA levels at baseline. Six patients (AC, $n=$ 2; TC, $n=4$ ) did not undergo any treatment over the 12-month follow-up. CgA levels were elevated in 1 patient at baseline. Levels remained elevated for this patient (AC tumor) but remained normal in the other 5 (Fig. 5a).

Sixteen of the $22 \mathrm{PD}$ patients (AC, $n=8$; TC, $n=8$ ) either started $(n=7)$ SSA therapy (Sandostatin LAR 20$30 \mathrm{mg})$ or continued $(n=9)$ with therapy. At baseline, 7 had elevated levels. SSA was associated with a decrease in $\mathrm{CgA}$ in only 1 patient (6\%, AC tumor). No changes were noted in 9. Elevation was evident in 6 (Fig. 5a, b). Spider plot analysis (Fig. 5b) identified that SSA was associated with a significant increase in $\mathrm{CgA}(p=0.0021 ; n=16)$ compared to those not treated $(n=6)$. This suggests the ineffectiveness of SSA in this cohort of PD patients.

Twenty-seven patients were considered stable at baseline. Eleven exhibited elevated CgA levels. Twenty-three patients (AC, $n=5$; TC, $n=18$ ) were not treated over the 12-month follow-up. In 6 patients, CgA decreased despite no treatment; was unchanged in 12 (was always normal $n=8$ or remained elevated $n=4)$ and increased in $5(22 \%$; Fig. 5 c). In the nine with elevated levels, 2 were PD. Four patients (AC, $n=1$; TC, $n=3$ ) treated with SSAs (Sandostatin LAR $20 \mathrm{mg}$ ) all remained clinically stable. Spider plot analysis (Fig. 5d) identified that SSA was not associated with a significant changes in $\operatorname{CgA}(p=0.84 ; n=4)$ compared to those not treated $(n=23)$. This suggests low effectiveness of SSA in this cohort of SD patients.

\section{Discussion}

When CgA was initially described as a useful NET biomarker, there was considerable enthusiasm [23]. More recent assessments have expressed concerns [24-26]. $\mathrm{CgA}$ is currently rated by NCCN guidelines as a category 3 test which is defined: "Based upon any level of evidence, there is major NCCN disagreement that the test is appropriate" [27], while European Neuroendocrine Tumour Society has specifically identified that there is "little or no 


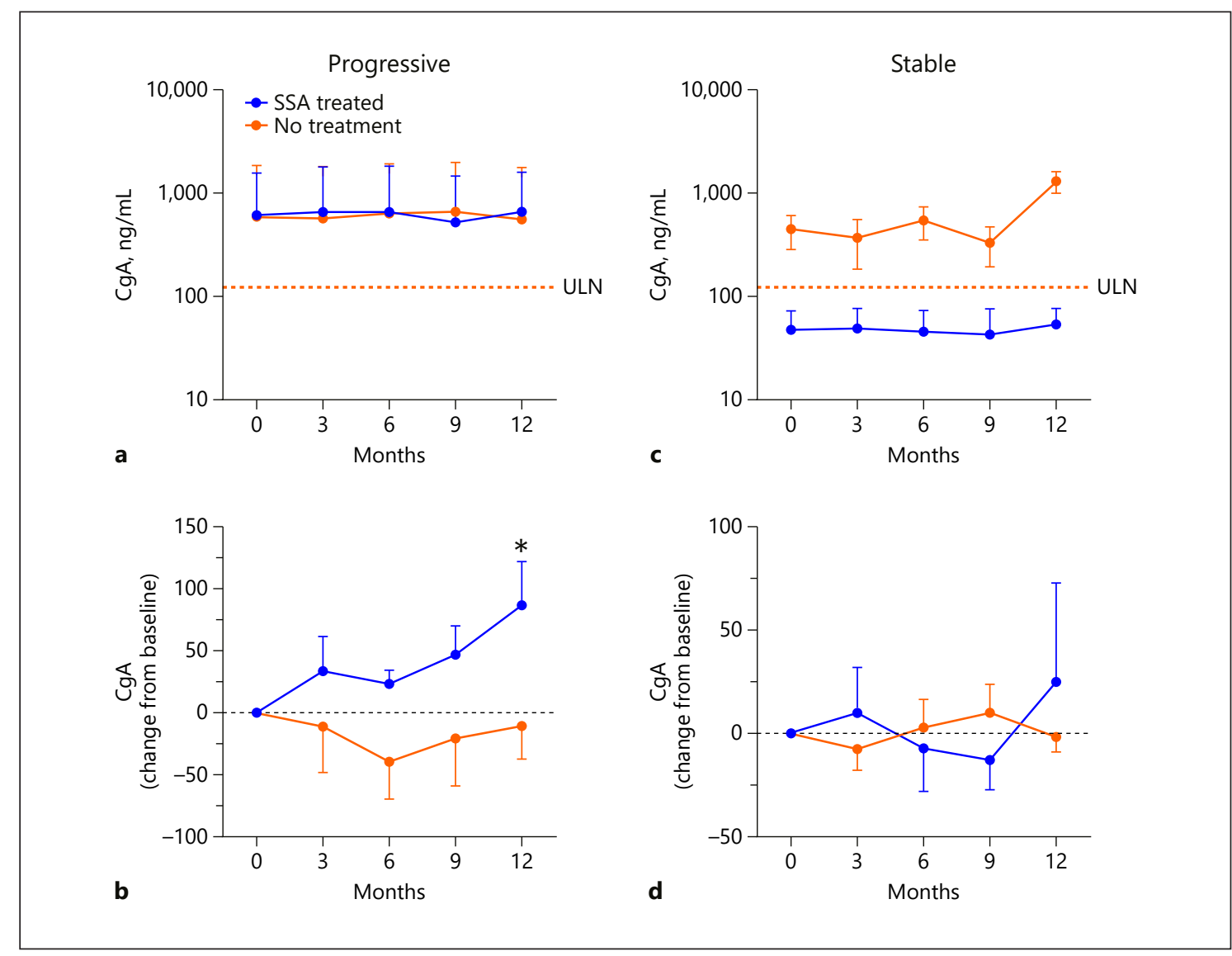

Fig. 5. Clinical utility of CgA and BPNET/NEN monitoring. a CgA levels of 22 patients with progressive disease either treated with SSA $(n=16)$ or not treated $(n=6)$ over a 12 -month period. $\mathbf{b}$ Spider plot analysis identified a significant difference (increase, $p=$ 0.0021) of SSA treatment on CgA. c CgA levels of 27 patients with
SD either treated with SSA $(n=4)$ or not treated $(n=23)$ over a 12-month period. $\mathbf{d}$ Spider plot analysis was unable to differentiate an impact of therapy ( $p=0.84)$ over the evaluation period. $\operatorname{CgA}$, chromogranin A. systematic empirical evidence" to use CgA for lung [12]. The paucity of BPNET/NEN biomarker studies [5], as well as the current CgA controversy, provided the impetus for a formal assessment of this biomarker in BPNET/ NEN disease. We evaluated the efficacy of CgA as a circulating biomarker in BPNET/NEN and compared it to controls, nonneuroendocrine lung diseases, BPNET/ NEN of different histological variants and grade, and during disease monitoring.

We identified that circulating chromogranin levels were clinically uninformative in the majority $(67 \%)$ of BPNET/NEN including those with extensive disease (only elevated in 43\%). Levels were also elevated in benign and other malignant lung disease. AUC analyses could not differentiate BPNET/NEN from other lung diseases or controls, and the overall accuracy metrics are significantly lower than those considered necessary for an effective biomarker [5]. Of more relevance to NETs, CgA measurement was incapable of differentiating histological variants (TC vs. AC) and exhibited no correlation with Ki-67. Similarly, both stable disease and progressive disease exhibited elevations in a similar proportion of patients (31-32\%). Monitoring BPNET/NEN over a 12 month period identified neither CgA levels per se nor changes in $\mathrm{CgA}$ were reflective of the natural history of the disease (progression).

"False"-positive levels were a feature of this study. These were identified in benign and malignant lung disease. In benign disease, this likely reflects increased numbers of pulmonary neuroendocrine cells compared to healthy controls [28] or the high prevalence of gastroesophageal reflux and the concomitant use of acid suppressive therapy $[29,30]$. A localized increase of neuroendocrine cells is well recognized to increase circulating 
chromogranin as noted in gastric carcinoids and ECL hyperplasia of the gastric fundus [31], while proton pump inhibitor treatment will induce an elevation in this biomarker regardless of short- or long-term use [32]. It is also possible that the concomitant underlying inflammatory processes in the lung that occur with COPD and IPF [33] may involve the diffuse neuroendocrine system and accentuate release of cytokines and neuropeptides [34].

More than $10 \%$ of ACC and SCC (16\%) also exhibited high levels. We suspect that there may be some degree of neuroendocrine differentiation in those with elevated levels [35] as has been previously noted [6], or inflammation may play a factor. Small cell lung cancers also exhibited elevated levels that are well-described [36] and likely reflect the histological observation that SCLC is of neuroendocrine origin, although poorly histologically differentiated and clinically distinct from carcinoids. SCLC is an aggressive disease with rapid progression and a marker that is unable to discern between SCLC and BPNET/NEN has obvious limitations given the significantly different clinical courses and management of the respective diseases, especially given the low survival rate of SCLC (5year survival rate: $<7 \%$ ) [37]. Overall, these data demonstrate that $\mathrm{CgA}$ is a poor diagnostic in lung neoplasia due to lack of specificity and also identify that elevated CgA levels are not exclusive to BPNET/NEN.

BPNET/NEN are classified into 2 histological variants (AC and TC) based upon biopsy, mitotic count, and areas of necrosis. The significance of correct identification of histology is heightened by the frequent metastases in AC $(>50 \%)$ as opposed to TC (25\%) [12]. The distinction between the AC and TC phenotype is crucial due to their substantially different clinical outcomes with 5 -year survival rates for $\mathrm{AC}$ being 50\% compared to $~ 88 \%$ in TC [38]. Additionally, there is controversy regarding the role of the Ki-67 proliferation index in differentiating AC (20\%) from TC 5\% [39]. CgA levels alone cannot distinguish the 2 histological variants. Of note, similar proportions of TC (30\%) and AC (36\%) exhibited an elevated CgA. AUC analysis alone could not differentiate between the 2 histological variants. We also could not identify any relationship between CgA levels and Ki-67 (grade) per se. This is consistent with 1 parameter, $\mathrm{CgA}$, functioning as a measure of secretion, and a second, Ki-67, as a measure of proliferation. These 2 parameters do not appear to therefore be linked.

Staging also was not associated with clinically useful alterations in CgA. While CgA is known to be higher in those with extensive, for example, stage IV GEP-NET disease [40] and we did identify Stage IV BPNET/NEN to have the highest levels, only $43 \%$ were elevated. Twentyeight percent of those with no metastases also exhibited elevated levels, while $33 \%$ of those with lymph node metastases were abnormal. This was not significantly different identifying that CgA may not be useful for staging BPNET/NEN.

We then, using RECIST 1.0 criteria, evaluated whether CgA levels could be a predictor of the patient's clinical status. Both progressive disease and stable disease had significantly higher CgA levels than controls. Levels were, however, not significantly elevated in PD compared with SD nor were the proportion of patients with elevated levels (31-32\%). It is not therefore surprising that the AUC analysis failed to differentiate the 2 cohorts. The detection of progressive disease in BPNET/NEN is of considerable clinical relevance in terms of management and outcome. The decision to utilize certain therapies and treatments, such as surgical resection or chemotheraputic drugs, is dictated by the identification of progressive versus stable disease [12]. Our data indicate that CgA levels are a poor determinant of PD and therefore cannot effectively facilitate therapeutic options.

We also examined the clinical utility of CgA measurement by monitoring levels in 49 patients over a 12-month period. Of the 49 patients, 22 were defined as progressive disease and 27 were stable at baseline measurements. No significant changes in CgA levels were detected irrespective of the patient's clinical status (SD vs. PD) during treatment, or their response to SSA treatment. Of the $27 \mathrm{pa}$ tients with stable disease at baseline, 6 patients developed progressive disease during follow-up. Only 2 out of the 6 patients that progressed showed an increase in CgA levels. Changes in CgA levels measured during follow-up were not related to $\mathrm{PD}$, or disease stabilization but were increased in a proportion of $\mathrm{PD}$ undergoing treatment. Although these data are limited because they were derived from small subgroups, they do suggest that CgA levels do not consistently reflect either responses to SSA therapy or function as an accurate Type 0 biomarker in BPNET/ NEN. Clearly, larger prospective studies are required.

As in any study, there are some limitations. These include that it was multicentric and therefore no central review of histology was undertaken, it was retrospective and some of the cohorts are small. It should be noted that multicentricity may be a positive feature since data from a number of institutions are representative of real-world information. The group sizes studied are also substantial for a relatively uncommon disease. The cohorts of stable and progressive disease evaluated over time are limited and therefore no definitive conclusions can be derived. 
However, the data support the general consensus that measurement of a single complex secretory protein cannot capture the complex biological behavior of a proliferative neoplasia [5].

There is a critical need for an effective circulating biomarker since currently only imaging can provide information regarding disease status. Imaging an indolent disease such as BPNET/NEN, however, has significant limitations in terms of costs, radiation exposure, and interpretation [41]. Somatostatin receptors (SSTR1, SSTR2A, SSTR5) expression has been investigated in paraffin-embedded BPNEN samples and reported to be of use as a diagnostic, prognostic, and therapeutic marker. Tissue expression levels measured by qRT-PCR may be capable of differentiating BPNET/NEN variants [42]. However, only limited investigation of these markers as circulating targets has been reported (e.g., SSTR2 and 5 detectable on CTCs) [43]. Other strategies reported include ELISA measurement of serum levels of VEGFR-1, which identified that VEGFR-1 levels were elevated and closely correlated with tumor size and metastasis [19]. These important results require further assessment in larger cohorts and an evaluation of whether discrimination between histological variants is feasible. Several miRNAs have been investigated. One study identified a set of 5 miRNAs (miR-129-5p, miR-409-3p, miR-409-5p, miR-185, miR497) that were differentially upregulated in TC versus AC BPCs [44]. A separate study reported that Hsa-miR-222-3p's putative target, $C D K N 1 B$, was overexpressed in TC compared to AC [45]. The critical limitation of these data is, however, that they are tissue assays and are not currently available as blood tests.

A recent multigene analysis study using whole-blood mRNA has evaluated a 51-NET transcript panel in 25 BPNET/NEN and 25 controls [46]. A multianalyte algorithm analysis technique reported the ability to differentiate BPNET/NEN from controls as well as stable from progressive disease with greater than $90 \%$ accuracy [46]. A subsequent follow-up study using the same technology demonstrated the assay had excellent diagnostic metrics (93\% sensitivity, $89 \%$ specificity) and could accurately differentiate progressive from stable disease (AUC 0.91) [47]. A separate independent study [48], in a large group of patients, confirmed the diagnostic utility of the multigene assay in BPNET/NEN and demonstrated its utility as a marker of progression. The clinical utility of this multi gene analysis (NETest) in the surgical management of BPNET/NEN noted the accuracy of the NETest to identify complete resection and detect residual disease or recurrence [47].
Our study demonstrates that circulating CgA levels are not clinically useful in the diagnosis of BPNET/NEN and have no clinical utility in monitoring disease management. This reflects growing awareness that measurement of monoanalyte biomarkers do not provide an accurate multidimensional view of the complex processes inherent in neoplasia. Although CgA levels were elevated compared to controls, two thirds of all BPNET/NEN patients had normal CgA levels. Assessment of the one-third that were abnormal demonstrated that $\mathrm{CgA}$ was incapable of distinguishing progressive from stable disease, failed to define histological variants, exhibited no relationship to grade, and could not be used to predict disease outcome. Moreover, an elevated CgA was commonly identified in benign lung diseases while other neoplasia, for example, NSCLC, also expressed high circulating levels.

$\mathrm{CgA}$ is currently considered a controversial biomarker, for GEP-NETs $[24,27]$ and in particular for BPNET/ NEN [12]. We are reminded of the insightful comments of Ovid in AD 1 - "Tempora mutantur, nos et mutamur in illis" (Times are changed; we, too, are changed within them) - these immemorial lines penned 2 thousand years ago confirm that previously valid observations may need to be altered with the passage of time. The current study confirms the clearly apparent lack of utility of CgA, a once useful general biomarker, whose efficacy has been supplanted by novel multianalyte genomic biomarkers. We respectfully suggest that further investigations should be undertaken in the evaluation of other markers such as molecular-based assays or alternative proliferative or metabolic regulators of bronchopulmonary NET disease.

\section{Disclosure Statement}

M.K. and S.M. are employees of Wren Laboratories. I.A.D. and I.M.M. have consulted for Wren Laboratories LLC. Wren Laboratories is involved in the development of the NETest and is licensed to use it. L.B. has received support from Advanced Accelerator Applications.

\section{Author Contributions}

All authors contributed equally to the manuscript. 


\section{References}

1 Henry NL, Hayes DF. Cancer biomarkers. Mol Oncol. 2012 Apr;6(2):140-6.

2 Strimbu K, Tavel JA. What are biomarkers? Curr Opin HIV AIDS. 2010 Nov;5(6):463-6.

3 i H, Cho JY; I H. Lung Cancer Biomarkers. Adv Clin Chem. 2015;72:107-70.

4 Duffy MJ. Use of Biomarkers in Screening for Cancer. Adv Exp Med Biol. 2015;867:27-39.

5 Oberg K, Modlin IM, De Herder W, Pavel M, Klimstra D, Frilling A, et al. Consensus on biomarkers for neuroendocrine tumour disease. Lancet Oncol. 2015 Sep;16(9):e435-46.

6 Travis WD, Brambilla E, Nicholson AG, Yatabe Y, Austin JH, Beasley MB, et al.; WHO Panel. The 2015 World Health Organization Classification of Lung Tumors: Impact of Genetic, Clinical and Radiologic Advances Since the 2004 Classification. J Thorac Oncol. 2015 Sep; 10(9):1243-60.

7 Rena O, Filosso PL, Ruffini E, Oliaro A. [Bronchopulmonary carcinoid tumours]. Minerva Chir. 2002 Aug;57(4):403-23.

8 Öberg K, Hellman P, Ferolla P, Papotti M; ESMO Guidelines Working Group. Neuroendocrine bronchial and thymic tumors: ESMO Clinical Practice Guidelines for diagnosis, treatment and follow-up. Ann Oncol. 2012 Oct;23(Suppl 7):vii120-3.

9 Ardill JE, O'Dorisio TM. Circulating biomarkers in neuroendocrine tumors of the enteropancreatic tract: application to diagnosis, monitoring disease, and as prognostic indicators. Endocrinol Metab Clin North Am. 2010 Dec;39(4):777-90.

10 Chong S, Lee KS, Chung MJ, Han J, Kwon OJ, Kim TS. Neuroendocrine tumors of the lung: clinical, pathologic, and imaging findings. Radiographics. 2006 Jan-Feb;26(1):41-57; discussion 57-8.

11 Kaifi JT, Kayser G, Ruf J, Passlick B. The Diagnosis and Treatment of Bronchopulmonary Carcinoid. Dtsch Arztebl Int. 2015 Jul; 112(27-28):479-85.

12 Caplin ME, Baudin E, Ferolla P, Filosso P, Garcia-Yuste M, Lim E, et al.; ENETS consensus conference participants. Pulmonary neuroendocrine (carcinoid) tumors: european Neuroendocrine Tumor Society expert consensus and recommendations for best practice for typical and atypical pulmonary carcinoids. Ann Oncol. 2015 Aug;26(8):1604-20.

13 Bottoni P, De Michele T, Scatena R. A Critical Approach to Clinical Biochemistry of Chromogranin A. Adv Exp Med Biol. 2015;867: $317-23$.

14 Modlin IM, Gustafsson BI, Moss SF, Pavel M, Tsolakis AV, Kidd M. Chromogranin A-biological function and clinical utility in neuro endocrine tumor disease. Ann Surg Oncol. 2010 Sep;17(9):2427-43.
15 Weiler R, Fischer-Colbrie R, Schmid KW, Feichtinger $\mathrm{H}$, Bussolati $\mathrm{G}$, Grimelius L, et al. Immunological studies on the occurrence and properties of chromogranin A and B and secretogranin II in endocrine tumors. Am J Surg Pathol. 1988 Nov;12(11):877-84.

16 Ardill JE, Erikkson B. The importance of the measurement of circulating markers in patients with neuroendocrine tumours of the pancreas and gut. Endocr Relat Cancer. 2003 Dec;10(4):459-62.

17 Glinicki P, Jeske W. Chromogranin A (CgA)the influence of various factors in vivo and in vitro, and existing disorders on it's concentration in blood. Endokrynol Pol. 2010 Jul-Aug; 61(4):384-7.

18 Oberg K, Krenning E, Sundin A, Bodei L, Kidd M, Tesselaar M, et al. A Delphic consensus assessment: imaging and biomarkers in gastroenteropancreatic neuroendocrine tumor disease management. Endocr Connect. 2016 Sep;5(5):174-87.

19 Telega A, Kos-Kudła B, Foltyn W, BlicharzDorniak J, Rosiek V. Selected neuroendocrine tumour markers, growth factors and their receptors in typical and atypical bronchopulmonary carcinoids. Endokrynol Pol. 2012; 63(6):477-82

20 Kidd M, Bodei L, Modlin IM. Chromogranin A: any relevance in neuroendocrine tumors? Curr Opin Endocrinol Diabetes Obes. 2016 Feb;23(1):28-37.

21 Ćwikła JB, Bodei L, Kolasinska-Ćwikła A, Sankowski A, Modlin IM, Kidd M. Circulating transcript analysis (NETest) in GEPNETs treated with Somatostatin Analogs defines Therapy. J Clin Endocrinol Metab. 2015 Nov;100(11):E1437-45.

22 Tukey J. Exploratory Data Analysis. AddisonWesley; 1997.

23 Eriksson B, Arnberg H, Oberg K, Hellman U, Lundqvist $\mathrm{G}$, Wernstedt $\mathrm{C}$, et al. Chromogranins-new sensitive markers for neuroendocrine tumors. Acta Oncol. 1989;28(3):325-9.

24 Marotta V, Zatelli MC, Sciammarella C, Ambrosio MR, Bondanelli M, Colao A, et al. Chromogranin A as circulating marker for diagnosis and management of neuroendocrine neoplasms: more flaws than fame. Endocr Relat Cancer. 2018 Jan;25(1):R11-29.

25 Marotta V, Nuzzo V, Ferrara T, Zuccoli A, Masone M, Nocerino L, et al. Limitations of Chromogranin A in clinical practice. Biomarkers. 2012 Mar; 17(2):186-91.

26 Molina R, Alvarez E, Aniel-Quiroga A, Borque $M$, Candás B, Leon A, et al. Evaluation of chromogranin A determined by three different procedures in patients with benign diseases, neuroendocrine tumors and other malignancies. Tumour Biol. 2011 Feb;32(1):13-22.

27 Shah MH, Goldner WS, Halfdanarson TR, Bergsland E, Berlin JD, Halperin D, et al. NCCN Guidelines Insights: Neuroendocrine and Adrenal Tumors, Version 2.2018. J Natl Compr Canc Netw. 2018 Jun;16(6):693-702.
28 Gu X, Karp PH, Brody SL, Pierce RA, Welsh MJ, Holtzman MJ, et al. Chemosensory functions for pulmonary neuroendocrine cells. Am J Respir Cell Mol Biol. 2014 Mar;50(3): 637-46.

29 Tobin RW, Pope CE 2nd, Pellegrini CA, Emond MJ, Sillery J, Raghu G. Increased prevalence of gastroesophageal reflux in patients with idiopathic pulmonary fibrosis. Am J Respir Crit Care Med. 1998 Dec;158(6): 1804-8.

30 Gribbin J, Hubbard R, Smith C. Role of diabetes mellitus and gastro-oesophageal reflux in the aetiology of idiopathic pulmonary fibrosis. Respir Med. 2009 Jun;103(6):927-31.

31 Eriksson B, Oberg K, Stridsberg M. Tumor markers in neuroendocrine tumors. Digestion. 2000;62(Suppl 1):33-8.

32 Pregun I, Herszényi L, Juhász M, Miheller P, Hritz I, Patócs A, et al. Effect of proton-pump inhibitor therapy on serum chromogranin a level. Digestion. 2011;84(1):22-8.

33 Balestro E, Calabrese F, Turato G, Lunardi F, Bazzan E, Marulli G, et al. Immune Inflammation and Disease Progression in Idiopathic Pulmonary Fibrosis. PLoS One. 2016 May; 11(5):e0154516.

34 Massironi S, Zilli A, Cavalcoli F, Conte D, Peracchi M. Chromogranin A and other enteroendocrine markers in inflammatory bowel disease. Neuropeptides. 2016 Aug;58:12734.

35 Gut P, Czarnywojtek A, Fischbach J, Bączyk $\mathrm{M}$, Ziemnicka K, Wrotkowska E, et al. Chromogranin A - unspecific neuroendocrine marker. Clinical utility and potential diagnostic pitfalls. Arch Med Sci. $2016 \mathrm{Feb} ; 12(1): 1-9$.

36 Petrović M, Bukumirić Z, Zdravković V, Mitrović S, Atkinson HD, Jurišić V. The prognostic significance of the circulating neuroendocrine markers chromogranin $\mathrm{A}$, pro-gastrin-releasing peptide, and neuron-specific enolase in patients with small-cell lung cancer. Med Oncol. 2014 Feb;31(2):823.

37 Byers LA, Rudin CM. Small cell lung cancer: where do we go from here? Cancer. 2015 Mar; 121(5):664-72.

38 Gustafsson BI, Kidd M, Chan A, Malfertheiner MV, Modlin IM. Bronchopulmonary neuroendocrine tumors. Cancer. 2008 Jul;113(1): 5-21.

39 Schnabel PA, Junker K. [Pulmonary neuroendocrine tumors in the new WHO 2015 classification: start of breaking new grounds?]. Pathologe. 2015 May;36(3):283-92.

40 Seregni E, Ferrari L, Bajetta E, Martinetti A, Bombardieri E. Clinical significance of blood chromogranin A measurement in neuroendocrine tumours. Ann Oncol. 2001;12(Suppl 2):S69-72. 
41 de Mestier L, Dromain C, d'Assignies G, Scoazec JY, Lassau N, Lebtahi R, Brixi H, Mitry E, Guimbaud R, Courbon F, d'Herbomez M, Cadiot G. Evaluating digestive neuroendocrine tumor progression and therapeutic responses in the era of targeted therapies: state of the art. Endocr Relat Cancer. 2014 Apr 28;21(3):R105-20.

42 Kaemmerer D, Specht E, Sänger J, Wirtz RM, Sayeg M, Schulz S, et al. Somatostatin receptors in bronchopulmonary neuroendocrine neoplasms: new diagnostic, prognostic, and therapeutic markers. J Clin Endocrinol Metab. 2015 Mar;100(3):831-40.
43 Childs A, Vesely C, Ensell L, Lowe H, Luong TV, Caplin ME, et al. Expression of somatostatin receptors 2 and 5 in circulating tumour cells from patients with neuroendocrine tumours. Br J Cancer. 2016 Dec;115(12): $1540-7$.

44 Rapa I, Votta A, Felice B, Righi L, Giorcelli J, Scarpa A, et al. Identification of MicroRNAs Differentially Expressed in Lung Carcinoid Subtypes and Progression. Neuroendocrinology. 2015;101(3):246-55.

45 Di Fazio P, Maass M, Roth S, Meyer C, Grups $\mathrm{J}$, Rexin $\mathrm{P}$, et al. Expression of hsa-let-7b-5p, hsa-let-7f-5p, and hsa-miR-222-3p and their putative targets HMGA2 and CDKN1B in typical and atypical carcinoid tumors of the lung. Tumour Biol. 2017 Oct; 39(10): 1010428317728417.
46 Kidd M, Modlin IM, Drozdov I, Aslanian H, Bodei L, Matar S, et al. A liquid biopsy for bronchopulmonary/lung carcinoid diagnosis. Oncotarget. 2017 Dec;9(6):7182-96.

47 Filosso PL, Kidd M, Roffinella M, Lewczuk A, Chung KM, Kolasinska-Cwikla A, et al. The utility of blood neuroendocrine gene transcript measurement in the diagnosis of bronchopulmonary neuroendocrine tumours and as a tool to evaluate surgical resection and disease progression. Eur J Cardiothorac Surg. 2018 Mar;53(3):631-9.

48 Malczewska A, Makulik K, Witkowska M, Bocian A, Walter A, Zajęcki W, et al. NETest liquid biopsy is diagnostic of small intestine and pancreatic neuroendocrine tumors and correlates with imaging. Endocr Connect. Forthcoming 2019. 